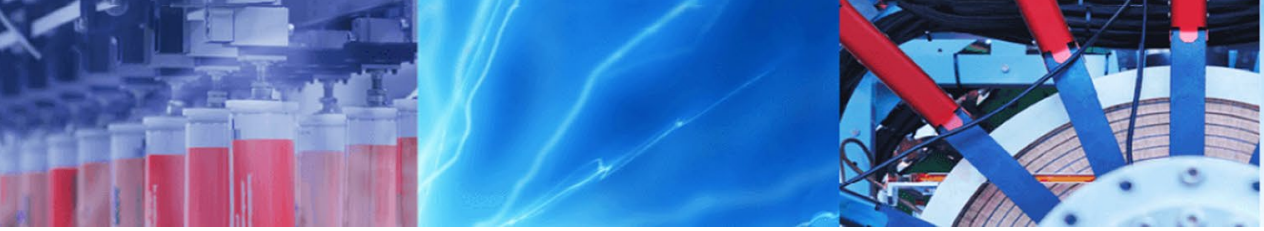

Research Article

\title{
Shifted Chebyshev polynomials based solution of partial differential equations
}

\author{
P. Karunakar ${ }^{1}$. S. Chakraverty ${ }^{1}$ \\ (c) Springer Nature Switzerland AG 2019
}

\begin{abstract}
This paper presents an efficient numerical method based on shifted Chebyshev polynomials for solving Partial Differential Equations (PDEs). In this method, a power series solution in terms of shifted Chebyshev polynomials has been chosen such that it satisfies the given conditions. Plugging this series solution into the given PDE and using appropriate collocation points a system of linear equations with unknown Chebyshev coefficients is obtained. Then, unknowns are found with the help of Gauss elimination or Newton's method. Next, different discretization patterns have also been considered to understand the behavior of the results depending upon the collocation points in the domain. These are the two main modifications and novelty of the procedure and this small contribution in the assumption of power series solution in terms of shifted Chebyshev polynomials results in obtaining the approximate solution with less number of terms with good accuracy. Several numerical examples are provided to confirm the reliability and effectiveness of the proposed method.
\end{abstract}

Keywords Shifted Chebyshev polynomials · Wave equation · Gauss elimination method · Collocation method

\section{Introduction}

The Partial Differential Equations (PDEs) attained a remarkable attention as many problems in science and engineering are modeled using PDEs. The analytical solution of the PDEs may not be found always due to the complexity of the differential equations. To overcome this shortcoming, method of characteristics, finite difference method [1, 2], finite element method [3-5], finite volume method [6, 7], and homotopy perturbation method [8-11] have been proven to be efficient and suitable to solve partial differential equations. With the aids of similarity variables, coupled partial differential equation can be converted to ordinary differential equation [12-15]. In recent years, Chebyshev polynomials acquired phenomenal attention for finding the solution of various types of PDEs like linear, non-linear, coupled system of PDEs and fractional differential equations. As such, Khalifa et al. [18] solved second and fourth order elliptic equations using spectral method based on
Chebyshev polynomials. Akyüz-Dascioglu [19] used Chebyshev polynomials for approximating the solution of high-order partial differential equations. A method based on shifted Chebyshev polynomials applied to find the numerical solution of fractional differential equations by Bhrawy et al. [20]. Caporale and Cerrato [21] used Chebyshev nodes to approximate partial differential equations. The solution of space fractional order diffusion equation was determined using second kind shifted polynomials by Sweilam et al. [22]. Mall and Chakraverty proposed a new algorithm using Chebyshev polynomials as base functions to solve singular initial value problems of Emden-Fowler type equations [23] and Lane-Emden type equations [24] by Chebyshev neural network method. Interested researchers are suggested to see [25-31] and therein for different methods to approximate the solution of various differential equation using Chebyshev polynomials.

The most common concept in the above discussed literature is that, initially a series solution in terms of Chebyshev

S.Chakraverty, sne_chak@yahoo.com; P. Karunakar, karunakarperumandla@yahoo.com | ${ }^{1}$ Department of Mathematics, National Institute of Technology Rourkela, Rourkela, Odisha 769 008, India.

SN Applied Sciences (2019) 1:285 | https://doi.org/10.1007/s42452-019-0292-z

Received: 9 January 2019 / Accepted: 21 February 2019 / Published online: 1 March 2019 
polynomials is assumed with unknown Chebysheve coefficients. Further, the unknown Chebysheve coefficients have been found using various techniques like spectral matrix method, artificial neural network etc. In the present paper, a power series solution has been chosen such that all the terms of the series satisfy the given conditions and then these are used in collocation method. Different discretization patterns have also been considered to understand the behavior of the results depending upon the collocation points in the domain. These are the main modifications and novelty of the procedure and this small contribution in the assumption of power series solution in terms of shifted Chebyshev polynomials results in obtaining the approximate solution with less number of terms with good accuracy.

Rest of the article is constructed as follows. In Sect. 2, basics properties of the Chebyshev polynomial have been reviewed. Description of the proposed method for linear PDEs is presented in Sect. 3. In Sect. 4, a few numerical examples are solved using the proposed method. The solutions are also compared with existing analytical results to show effectiveness of the method. The paper ends with Sect. 5, by concluding the remarks.

\section{Preliminaries of Chebyshev polynomials}

In this section basic and some important properties of Chebyshev polynomials are presented.

Russian Mathematician Pafnuty Chebyshev introduced nth degree polynomial with leading coefficient unity in the interval $[-1,1]$, in his paper entitled 'Théorie des mécanismes connus sous le nom de parallélogrammes' in the year 1854 as below [16]

$\frac{1}{2^{n-1}} \cos \left(n \cos ^{-1}(x)\right)=\frac{1}{2^{n-1}} T_{n}(x)$

The Chebyshev polynomials may be determined using the recurrence formula [22]

$T_{n+1}(x)=2 x T_{n}(x)-T_{n-1}(x), \quad T_{0}(x)=1, \quad T_{1}(x)=x, \quad n=1,2,3, \ldots$

First four Cheyshev polynomials are given by

$$
\begin{aligned}
& T_{0}(x)=1 \\
& T_{1}(x)=x \\
& T_{2}(x)=2 x^{2}-1 \\
& T_{3}(x)=4 x^{3}-3 x
\end{aligned}
$$

Further, the Chebyshev polynomials are defined in the interval $[0,1]$ by introducing the change in the variable $x=2 t-1$ [22] are called Shifted Chebyshev polynomials and are defined as

$T_{n}^{*}=T_{n}(2 t-1), \quad n=0,1,2, \ldots$
The first few shifted Cheyshev polynomials are

$T_{0}(t)=1$

$T_{1}(t)=2 t-1$

$T_{2}(t)=1+8 t^{2}-8 t$

$T_{4}(x)=32 t^{3}-48 t^{2}+18 t-1$

which all are defined in $t \in[0,1]$.

\section{Shifted Chebyshev collocation method}

This section describes the method of finding the solution of partial differential equations using shifted Chebyshev polynomials. To illustrate the present method, we consider general partial differential equation with two independent variables $x$ and $y$ and dependent variable $u$ as

$A(u)=f(x, y)$

subject to the conditions: $u\left(x_{0}, y\right)=g(y)$ and/or $u\left(x, y_{0}\right)=h(x)$

where $u$ is unknown function; $f, g$ and $h$ are known functions and, $x_{0}$ and $y_{0}$ are constants.

First, we assume a bivariate power series solution in terms of shifted Chebyshev polynomials that satisfies conditions given in Eq. (5) as below

$u(x, y)=\phi(x, y)+\psi(x, y) \sum_{r=0}^{N} \sum_{s=0}^{N} a_{r s} T_{r}^{*}(x) T_{s}^{*}(y)$

It is worth mentioning that $\phi(x, y)$ and $\psi(x, y)$ are functions of $x$ and $y$ which are to be chosen such that Eq. (6) satisfies the given conditions (5).

Next, plugging assumed solution (6) in Eq. (4), we get a residual equation $R(x, y)=0$, which involves chebyshev coefficients $a_{r s}$ and using proper collocation points in the domain $[0,1] \times[0,1]$, we get system of equation with unknowns Chebyshev coefficients $a_{r s}$ as

$A v=b$

where $A$ is the known coefficient matrix, $v$ is the column vectors with Chebyshev coefficients and $b$ is the known right hand side vector. Here $A$ and $b$ will have real entries which are obtained using collocation points in the residual equation $R(x, y)=0$. Here $N_{p}^{2}$ collocation points are needed if we consider $N_{p}$ polynomials where $N_{p}=N+1$. Finally solving the system of equation one may get the Chebyshev coefficients $a_{r s}$ 's and substituting these in Eq. (6) we get the approximate solution of (4).

It may be noted that discretization of domain is a critical issue. As such, various discretization patterns are also tried and finally the best one is chosen for obtaining acceptable results. 


\section{Numerical results and discussion}

In this section, three example problems have been solved using the present method.

Example 1 First we consider the Cauchy problem for the one dimensional homogenous wave equation [17]

$u_{y y}=u_{x x}, \quad 0 \leq x \leq 1, \quad 0 \leq y \leq 1$

$u(x, 0)=x^{3} \quad$ and $\quad u_{y}(x, 0)=x$
The exact solution of the above differential equation is $u(x, y)=x^{3}+3 x y^{2}+x y$. Let us assume the bivariate series solution of wave Eq. (8) as below

$u(x, y)=\left(x^{3}+x y\right)+x y^{2} \sum_{r=0}^{2} \sum_{s=0}^{2} a_{r s} T_{r}^{*}(x) T_{s}^{*}(y)$

here $\phi(x, y)=x^{3}+x y$ and $\psi(x, y)=x y^{2}$. The assumed solution (10) satisfies initial conditions (9). Differentiating (10) with respect to $x$ and $y$ partially we obtain partial derivatives as

$$
\begin{aligned}
u_{x x}= & 6 x+4 a_{10} y^{2}+4 a_{11}\left(2 y^{3}-y^{2}\right)+4 a_{12}\left(8 y^{4}-8 y^{3}+y^{2}\right)+a_{20}(48 x-16) y^{2} \\
& +4 a_{21}(48 x-16)\left(2 y^{3}-y^{2}\right)+4 a_{22}(48 x-16)\left(8 y^{4}-8 y^{3}+y^{2}\right)
\end{aligned}
$$

and

$$
\begin{aligned}
u_{y y}= & 2 a_{00} x+a_{10} x(12 y-2)+a_{02} x\left(96 y^{2}-48 y+2\right)+2 a_{10}\left(2 x^{2}-x\right)+a_{11}\left(2 x^{2}-x\right)(12 y-2) \\
& +a_{12}\left(2 x^{2}-x\right)\left(96 y^{2}-48 y+2\right)+2 a_{20}\left(8 x^{3}-8 x^{2}+x\right)+a_{21}\left(8 x^{3}-8 x^{2}+x\right)(12 y-2) \\
& +a_{22}\left(8 x^{3}-8 x^{2}+x\right)\left(96 y^{2}-48 y+2\right)
\end{aligned}
$$

$$
\begin{aligned}
& \left\{6 x+4 a_{10} y^{2}+4 a_{11}\left(2 y^{3}-y^{2}\right)+4 a_{12}\left(8 y^{4}-8 y^{3}+y^{2}\right)+a_{20}(48 x-16) y^{2}+4 a_{21}(48 x-16)\left(2 y^{3}-y^{2}\right)\right. \\
& \left.\quad+4 a_{22}(48 x-16)\left(8 y^{4}-8 y^{3}+y^{2}\right)\right\}-\left\{2 a_{00} x+a_{10} x(12 y-2)+a_{02} x\left(96 y^{2}-48 y+2\right)\right. \\
& \quad+2 a_{10}\left(2 x^{2}-x\right)+a_{11}\left(2 x^{2}-x\right)(12 y-2)+a_{12}\left(2 x^{2}-x\right)\left(96 y^{2}-48 y+2\right)+2 a_{20}\left(8 x^{3}-8 x^{2}+x\right) \\
& \left.\quad+a_{21}\left(8 x^{3}-8 x^{2}+x\right)(12 y-2)+a_{22}\left(8 x^{3}-8 x^{2}+x\right)\left(96 y^{2}-48 y+2\right)\right\}=0
\end{aligned}
$$

Plugging Eqs. (11) and (12) into (8) we get the residual equations $R(x, y)=0$ as

Then using the nine collocation points, (since $N=2$ so number of collocation points required here is 9) in the domain $[0,1] \times[0,1]$ as given in Fig. 1 , we may obtain the system of nine linear equations as below

$$
\begin{aligned}
0 a_{00} & +0 a_{01}+0 a_{02}+1 a_{10}+0 a_{11}-1 a_{12}-4 a_{20}+0 a_{21}+16 a_{22}=0 \\
& -\frac{1}{2} a_{00}-\frac{1}{4} a_{01}+1 a_{02}+\frac{1}{2} a_{10}+0 a_{11}-\frac{3}{8} a_{12}-\frac{1}{4} a_{20}+\frac{17}{8} a_{21}+\frac{3}{2} a_{22}=-\frac{3}{2} \\
& -1 a_{00}+1 a_{01}-1 a_{02}+0 a_{10}+0 a_{11}+1 a_{12}+0 a_{20}-9 a_{21}+9 a_{22}=-3 \\
& -\frac{1}{2} a_{00}-\frac{7}{4} a_{01}-5 a_{02}-\frac{5}{2} a_{10}+2 a_{11}+\frac{13}{8} a_{12}-\frac{9}{4} a_{20}-\frac{9}{8} a_{21}+\frac{9}{2} a_{22}=-\frac{3}{2} \\
& -1 a_{00}-2 a_{01}-1 a_{02}+1 a_{10}+0 a_{11}+0 a_{12}+2 a_{20}+2 a_{21}-7 a_{22}=-3 \\
& -\frac{3}{2} a_{00}-\frac{3}{4} a_{01}+3 a_{02}-\frac{1}{2} a_{10}-\frac{1}{2} a_{11}+\frac{17}{8} a_{12}+\frac{5}{4} a_{20}-\frac{77}{8} a_{21}-\frac{23}{2} a_{22}=-\frac{9}{2} \\
& -1 a_{00}-5 a_{01}-25 a_{02}+4 a_{10}+4 a_{11}+5 a_{12}+8 a_{20}+13 a_{21}+33 a_{22}=-3 \\
& -\frac{3}{2} a_{00}-\frac{21}{4} a_{01}-15 a_{02}+\frac{3}{2} a_{10}-\frac{3}{2} a_{11}-\frac{63}{8} a_{12}+\frac{45}{4} a_{20}-\frac{101}{8} a_{21}-\frac{5}{2} a_{22}=-\frac{9}{2} \\
& -2 a_{00}-4 a_{01}-2 a_{02}-1 a_{10}-4 a_{11}-5 a_{12}+8 a_{20}-4 a_{21}-34 a_{22}=-6
\end{aligned}
$$




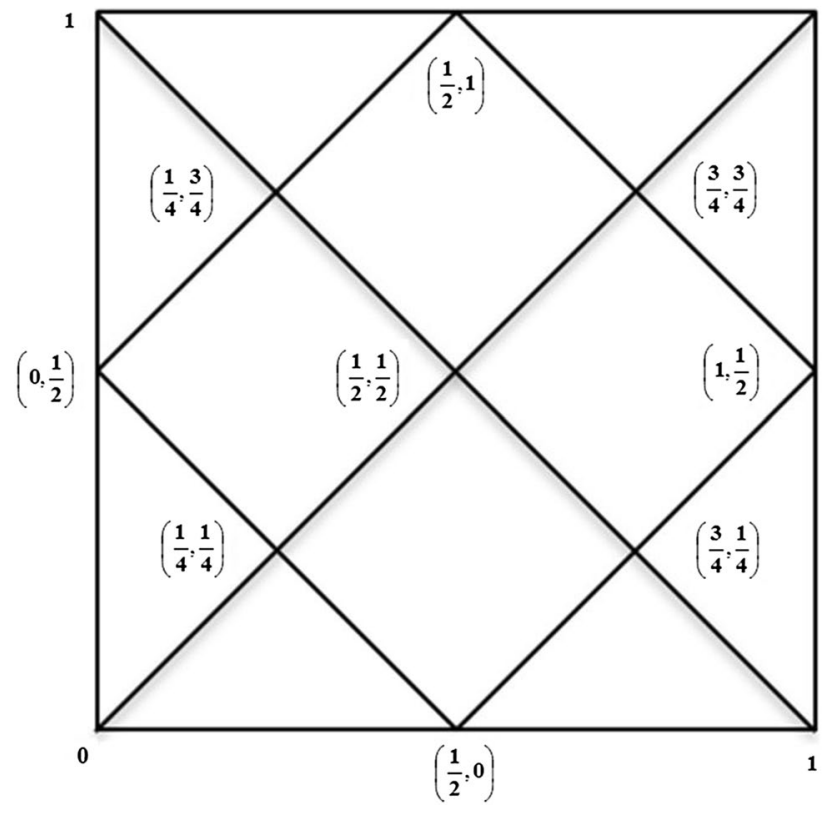

Fig. 1 Collocation points in the domain $[0,1]$

Solving the above system using Gauss elimination we get

$a_{00}=3 ; a_{01}=a_{02}=a_{10}=a_{11}=a_{12}=a_{20}=a_{21}=a_{22}=0$

Substituting all $a_{r s}^{\prime} s$ in Eq. (10) we obtain the solution of the wave Eq. (8) as $u(x, y)=x^{3}+3 x y^{2}+x y$, which is the exact solution. Figure 2 shows the solution of Eq. (8) in the domain $0 \leq x \leq 1,0 \leq y \leq 1$.

It is worth mentioning that choosing a proper discretization scheme of the domain plays an important role in obtaining the best approximate solution of the PDEs. To explain this, we have used two different discretization schemes in the next example.

Example 2 Let us now consider another example of partial differential equation of second order with variable coefficients [17]

$u_{y y}-\frac{1}{2} x^{2} u_{x x}=0, \quad 0 \leq x \leq 1, \quad 0 \leq y \leq 1$

$u(x, 0)=x \quad$ and $\quad u_{y}(x, 0)=x^{2}$

The exact solution of (13) is $u(x, y)=x+x^{2} \sinh (y)$ [17].

First we assume a solution of second order hyperbolic partial differential Eq. (13) that satisfies the given conditions (14) as below

$u(x, y)=\left(x+x^{2} y\right)+y^{2} \sum_{r=0}^{1} \sum_{s=0}^{1} a_{r s} T_{r}^{*}(x) T_{s}^{*}(y)$

Substituting the assumed series solution (15) in Eq. (13) we may get residue as below

$$
\begin{aligned}
& \left(2 a_{00}+a_{01}(6 y-2)\right)+2 a_{10}(2 x-1) \\
& \quad+a_{11}(2 x-a)(12 y-2)-0.5 x^{2}(1+2 y)=0
\end{aligned}
$$

Fig. 2 Solution of Eq. (8) in the domain $0 \leq x \leq 1,0 \leq y \leq 1$

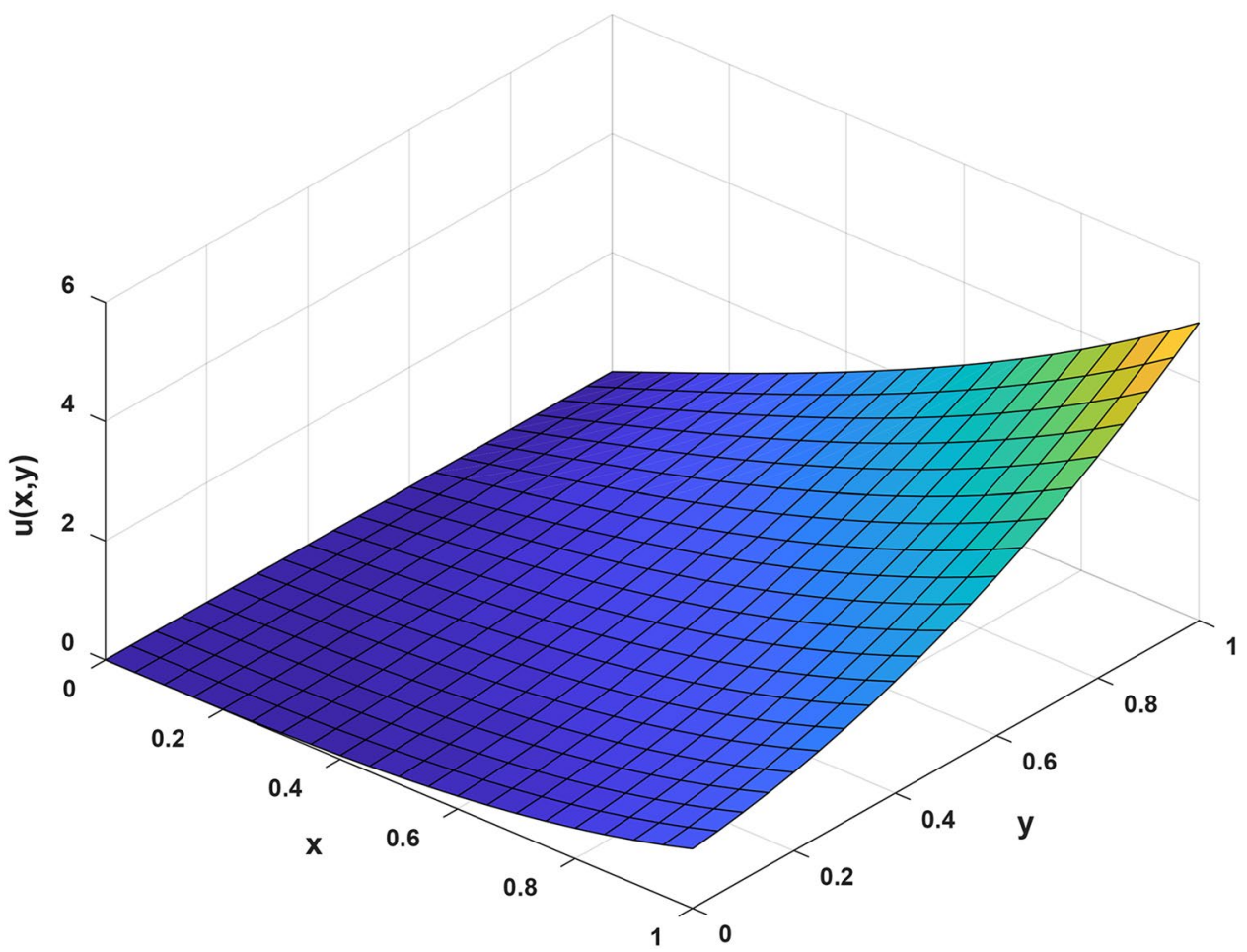




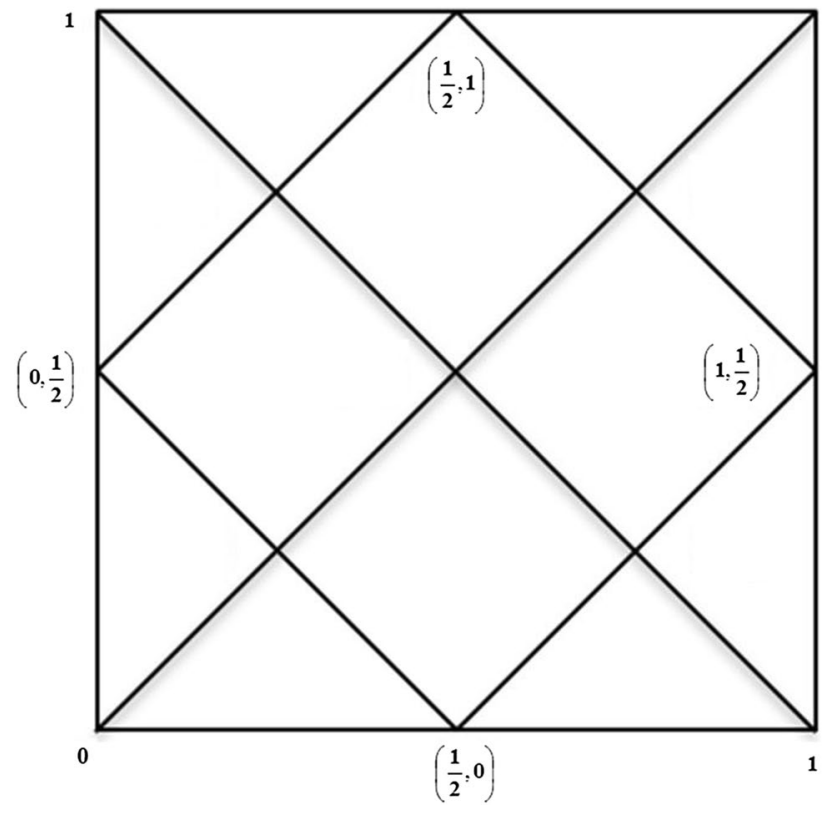

Fig. 3 Collocation points in the domain

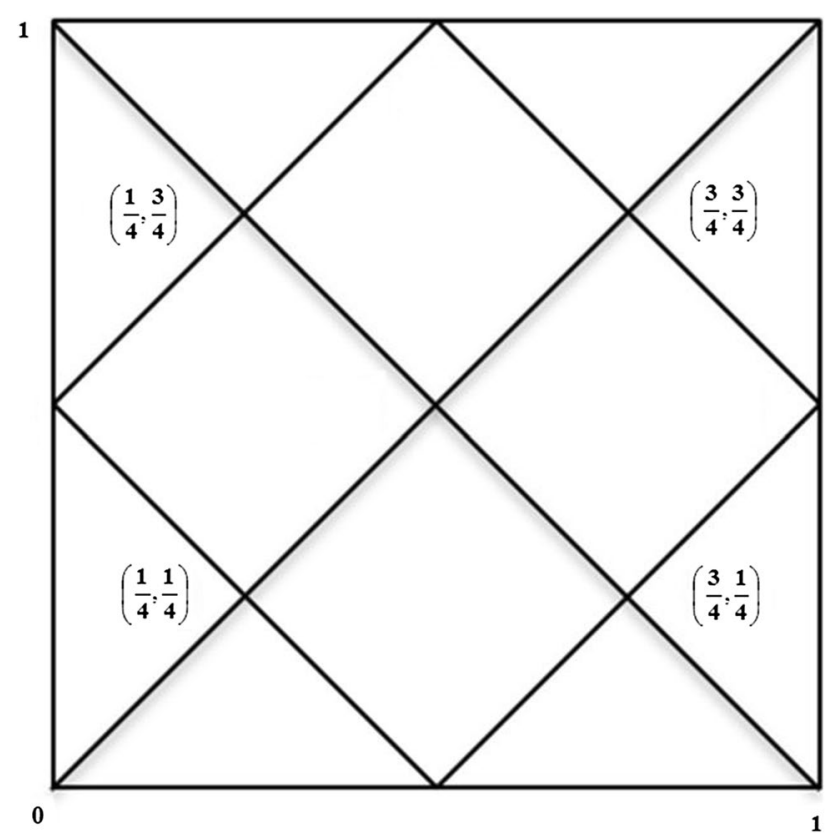

Fig. 4 Collocation points in the domain

here $N=1$ so using 4 collocation points $\left(\frac{1}{2}, 0\right),\left(0, \frac{1}{2}\right),\left(1, \frac{1}{2}\right)$ and $\left(\frac{1}{2}, 1\right)$ in the domain $[0,1] \times[0,1]$ as shown in the Fig. 3 we obtain the system of four linear equations as below
$2 a_{00}+0 a_{01}+0 a_{10}-2 a_{11}=\frac{1}{8}$

$2 a_{00}+a_{01}-2 a_{10}-4 a_{11}=0$

$2 a_{00}+2 a_{01}+0 a_{10}+0 a_{11}=\frac{3}{8}$

$2 a_{00}+a_{01}+2 a_{10}+4 a_{11}=1$

The above linear system of equation has no solution.

Now, we consider the collocation points $\left(\frac{1}{4}, \frac{1}{4}\right),\left(\frac{3}{4}, \frac{1}{4}\right),\left(\frac{1}{4}, \frac{3}{4}\right)$ and $\left(\frac{3}{4}, \frac{3}{4}\right)$ as shown in Fig. 4 , and substituting these collocation points in the residual Eq. (16) one may obtain the system of four linear equations as below

$2 a_{00}-\frac{1}{2} a_{01}-a_{10}-\frac{1}{2} a_{11}=\frac{3}{64}$

$2 a_{00}-\frac{1}{2} a_{01}+a_{10}+\frac{1}{2} a_{11}=\frac{27}{64}$

$2 a_{00}+\frac{5}{2} a_{01}-a_{10}-\frac{7}{2} a_{11}=\frac{5}{64}$

$2 a_{00}+\frac{1}{2} a_{01}+a_{10}+\frac{7}{2} a_{11}=\frac{5}{64}$

Solving the above linear system of equation using Guass elimination method we obtain the unknown coefficients of the series solution (15) as

$a_{00}=0.1042 ; \quad a_{01}=-0.0521 ; \quad a_{10}=0.2188 ; \quad a_{11}=-0.0625$ Hence the solution of the hyperbolic PDE (14) is

$$
\begin{aligned}
u(x, y)= & \left(x+x^{2} y\right)+y^{2}[0.1042-0.0521(2 y-1) \\
& +0.2188(2 x-1)-0.0625(2 x-1)(2 y-1)]
\end{aligned}
$$

Figure 5 represents the comparison of present solution of Eq. (13) with analytical solution for fixed $y=1$ and $N=1$, whereas Fig. 6 represents for $N=3$. The solution of Eq. (13) using the present method for $N=1$ and $N=3$ is compared with analytical solution for fixed $x=0.5$ in Fig. 7 .

It may be seen from Figs. 5 and 6 that the solution obtained by proposed method is almost same as exact solution just by considering $N=1$ and $N=3$ (that is two and four terms respectively), whereas in Ref. [17], $N=7$ has been taken to find the solution with error $6.0 \mathrm{E}-5$. From Fig. 7, it may be observed that for $N=1$ the solution of Eq. (13) by present method is having more error. But for $N=3$, the solution is matching with analytical solution. This shows that as $N$ 


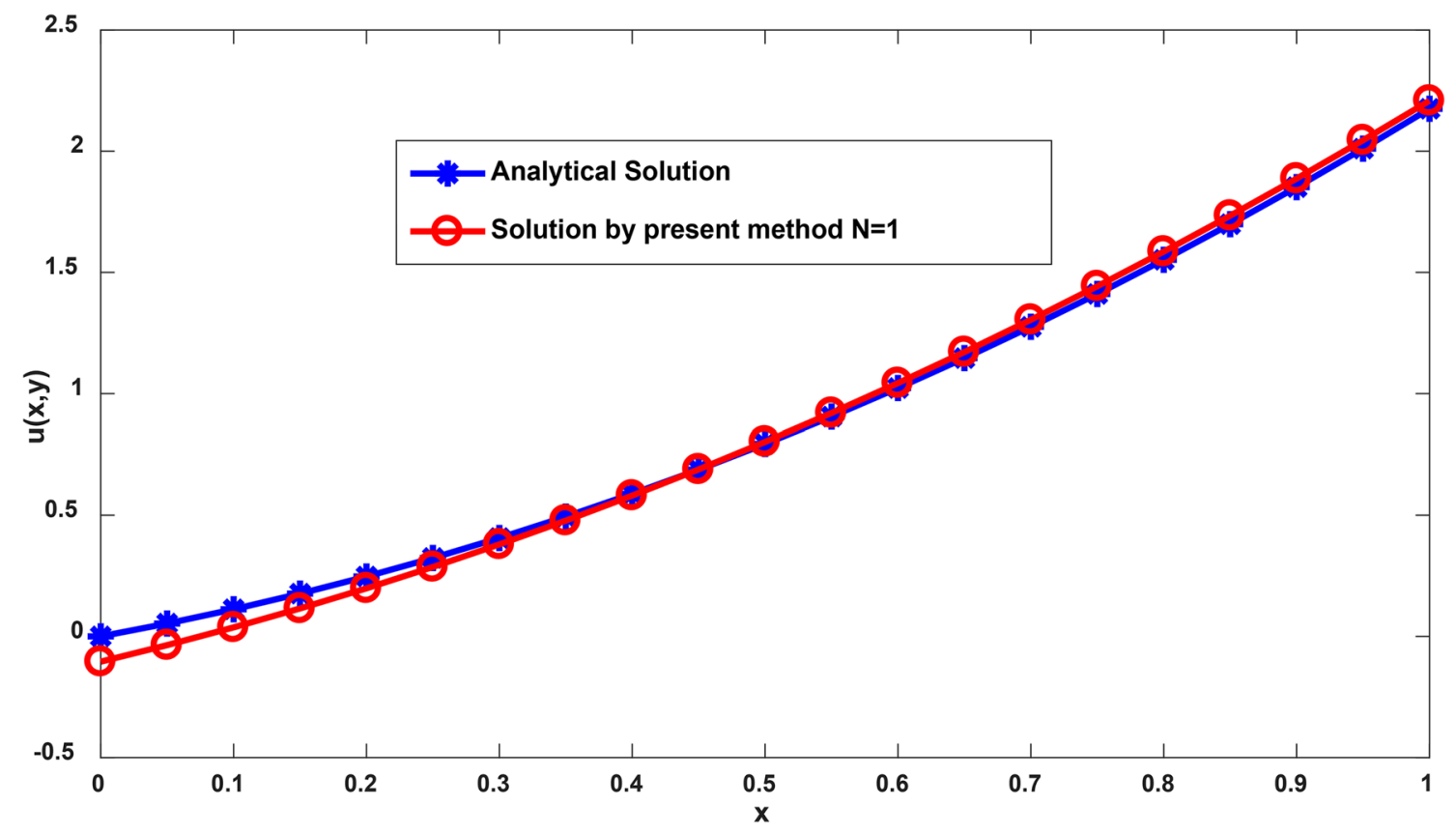

Fig. 5 Comparison of the solution of (13) by the proposed method with analytical solution at $y=1.0$ with $N=1$

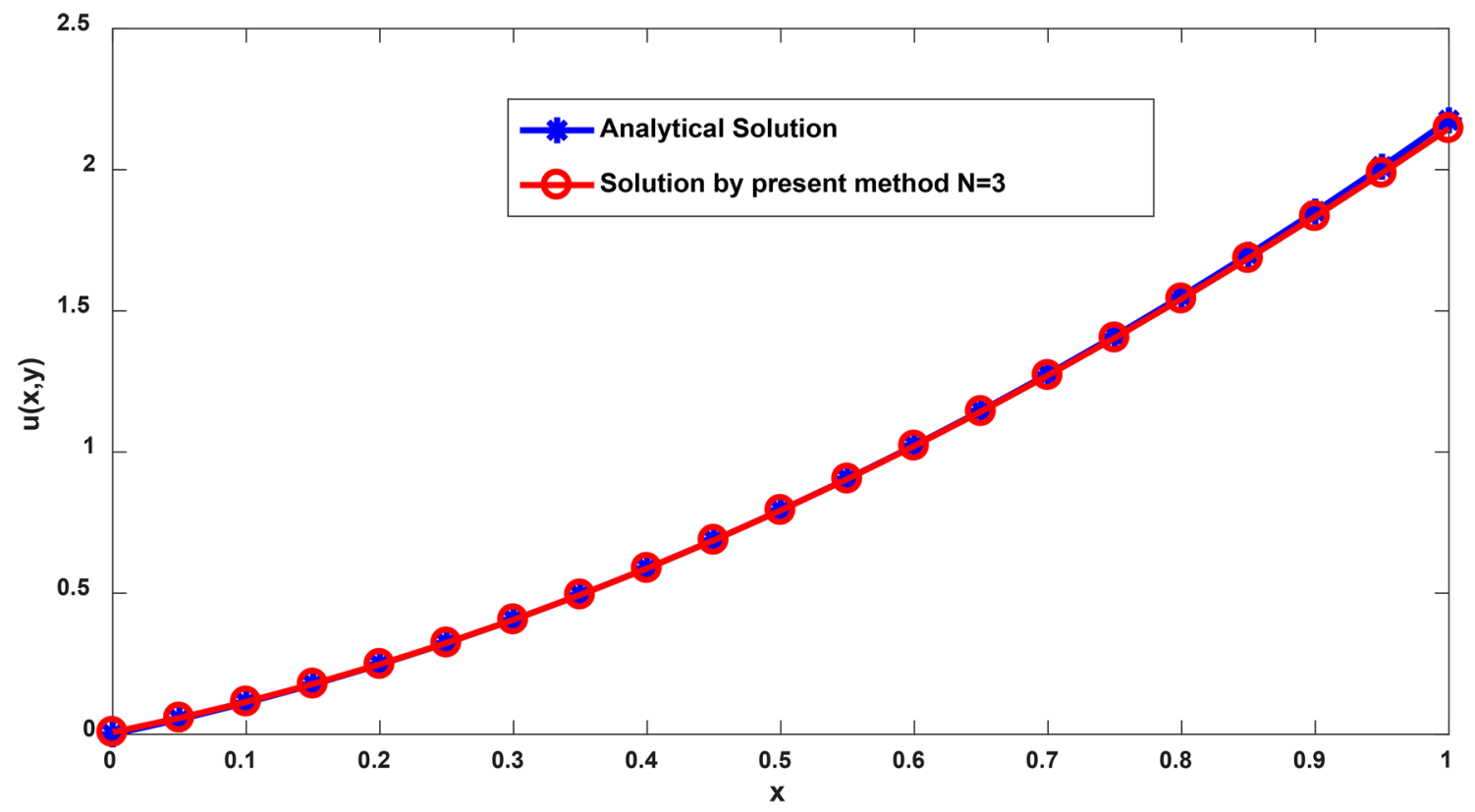

Fig. 6 Comparison of the solution of (13) by the proposed method with analytical solution at $y=1.0$ with $N=3$

increases the error in the solution by the present method will be less and it may exactly match with the analytical solution.

Example 3 Finally in order to show the efficacy of the present method, we consider another example of linear PDE

$u_{t t}-9 u_{x x}=e^{x}-e^{-x}$ subject to the conditions:

$u(x, 0)=x \quad$ and $\quad u_{t}(x, 0)=\sin (x)$

The exact solution of (17) using D'Alembert's formula is $u(x, t)=x+\frac{1}{3} \sin (x) \sin (3 t)-\frac{2}{9} \sinh (x)+\frac{2}{9} \sinh (x) \cosh (3 t)$ 


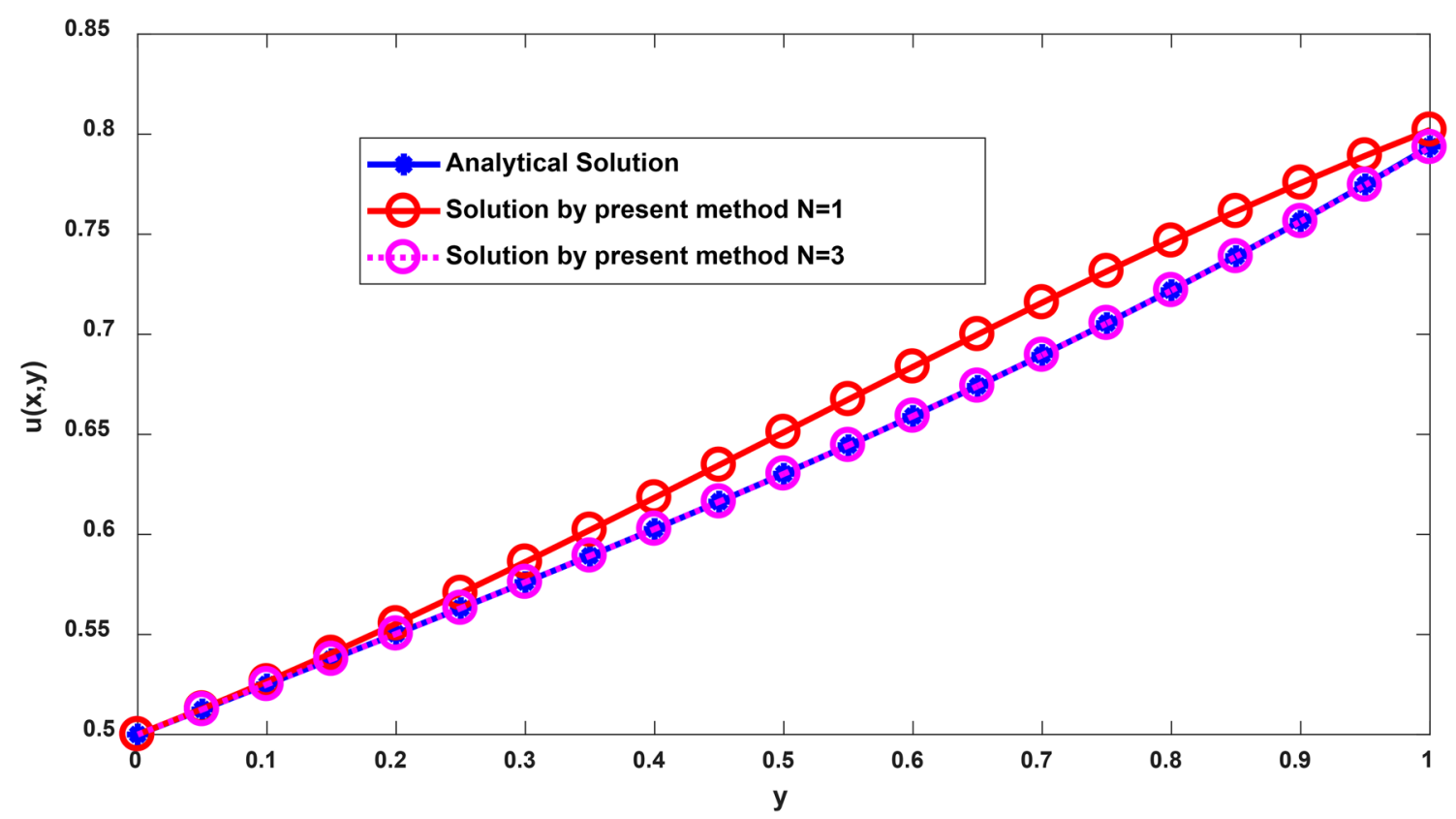

Fig. 7 Comparison of the solution of (13) by the proposed method with analytical solution at $x=0.5$ with $N=1$ and $N=3$

Let us assume the solution of the PDE (17) in the series form using shifted Chebyshev polynomials as below

$u(x, t)=x+t \sin (x)+x t^{2} \sum_{r=0}^{3} \sum_{s=0}^{3} a_{r s} T_{r}^{*}(x) T_{s}^{*}(y)$

Plugging the series solution (20) in Eq. (17) and proceeding like previous examples using collocation points given in Fig. 8, we get unknown coefficients
From Fig. 9, it can be seen that the approximate solution by the present method for $N=3$ (that is 4 terms), is in good agreement with the exact solution given in Eq. (19).

Various types of collocation points have been used. In example 1 four collocation points are on the boundary and remaining five points are inner. Using the proposed method and considering these collocation points the solution of the PDE is matching with exact solution. In example 2, two types of collocation points have been considered. Solution using

$a_{00}=0.8599, a_{01}=-0.0043, a_{02}=0.2183, a_{03}=0.0178, a_{10}=0.1471, a_{11}=0.0578, a_{12}=-0.0021, a_{13}=0.00052318$,

$a_{20}=0.0369, a_{21}=0.0141, a_{22}=-0.00027053, a_{23}=0.000055805, a_{30}=0.000061646, a_{31}=-0.00018182$,

$a_{32}=0.00010871, a_{33}=-0.000010631$

The solution of the PDE (17) is

collocation points on the boundary does not exist. But using the inner collocation points, the solution is in good agreement with exact solution. Finally in Example 3, mixed type

$$
\begin{aligned}
u(x, t)= & x+\sin (x)+x t^{2}\left\{0.8599-0.0043(2 t-1)+0.2183\left(8 t^{2}-8 t+1\right)+0.0178\left(32 t^{3}-48 t^{2}+18 t-1\right)\right. \\
& +0.1471(2 x-1)+0.0578(2 x-1)(2 t-1)-0.0021(2 x-1)\left(8 t^{2}-8 t+1\right)+0.00052318(2 x-1)\left(32 t^{3}-48 t^{2}+18 t-1\right) \\
& +0.0369\left(8 x^{2}-8 x+1\right)+0.0141\left(8 x^{2}-8 x+1\right)(2 t-1)-0.00027053\left(8 x^{2}-8 x+1\right)\left(8 t^{2}-8 t+1\right) \\
& +0.000055805\left(8 x^{2}-8 x+1\right)\left(32 t^{3}-48 t^{2}+18 t-1\right)+0.000061646\left(32 x^{3}-48 x^{2}+18 x-1\right) \\
& -0.00018182\left(32 x^{3}-48 x^{2}+18 x-1\right)(2 t-1)+0.00010871\left(32 x^{3}-48 x^{2}+18 x-1\right)\left(8 t^{2}-8 t+1\right) \\
& \left.-0.000010631\left(32 x^{3}-48 x^{2}+18 x-1\right)\left(32 t^{3}-48 t^{2}+18 t-1\right)\right\}
\end{aligned}
$$




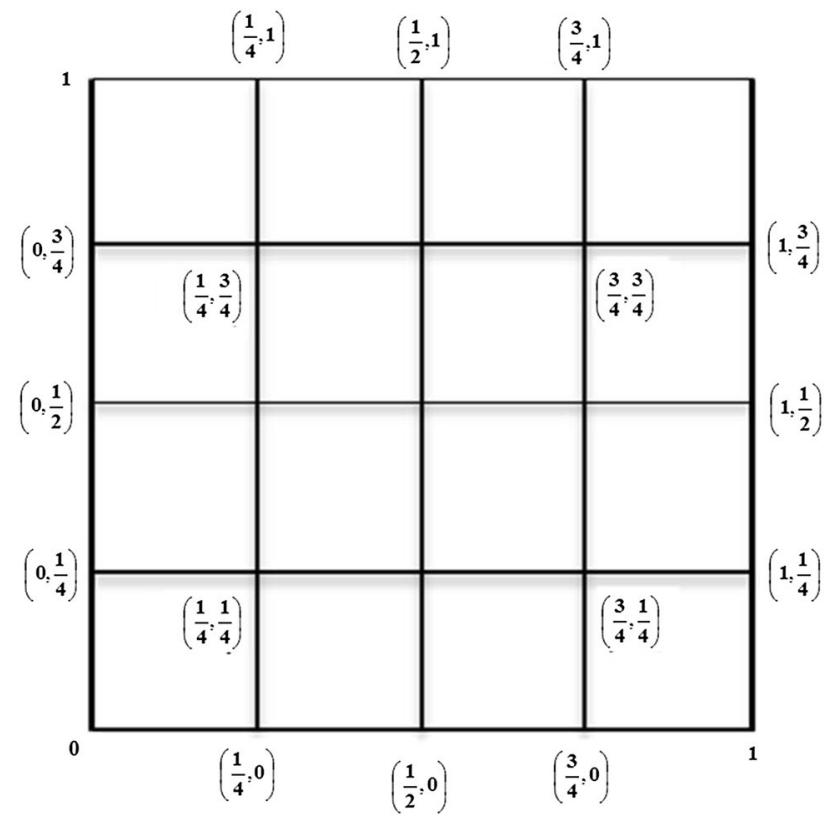

Fig. 8 Collocation points in the domain collocation points have been considered by which solution is in good agreement with the analytical solution. From this analysis it may be concluded that consideration of collocation points may vary for problem to problem.

\section{Conclusion}

The proposed modification in the collocation method based on shifted Chebyshev polynomial is successfully applied to solve different linear partial differential equations. In this method a series solution in terms of shifted Chebyshev polynomials is assumed satisfying the given conditions. This small modification in the assumption has succeeded to get the approximate solution with less number of terms comparing with other methods available in literature. Three examples have been solved and results are compared with existing literature. The obtained results confirm that the proposed method is effective and needs less number of terms to get the convergence.

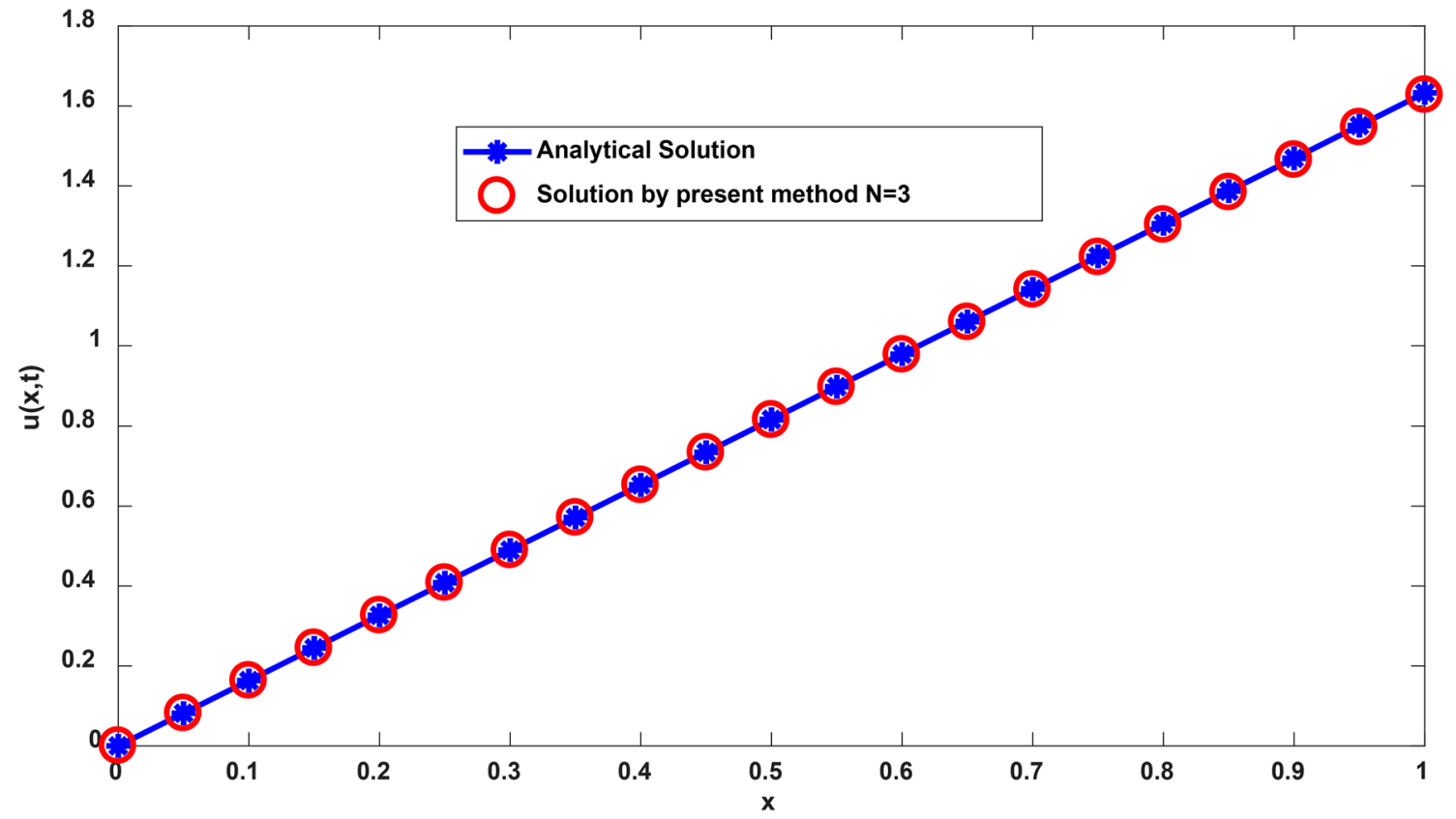

Fig. 9 Comparison of the solution of (17) by the present method for $N=3$ with analytical solution (19) at $t=0.5$ 


\section{Compliance with ethical standards}

Conflict of interest The authors declare that they have no conflict of interest.

\section{References}

1. Forsythe GW, Wasow WR (1960) Finite-difference methods for partial differential equations. Applied Mathematical Series Wiley, New York

2. Thomas JW (2013) Numerical partial differential equations: finite difference methods. Springer, New York

3. Johnson C (2012) Numerical solution of partial differential equations by the finite element method. Courier Corporation, Chelmsford

4. Alieldin SS, Alshorbagy AE, Shaat M (2011) A first-order shear deformation finite element model for elastostatic analysis of laminated composite plates and the equivalent functionally graded plates. Ain Shams Eng J 2:53-62

5. Nayak S, Chakraverty S (2018) Interval finite element method with MATLAB. Academic Press, New York

6. Eymard R, Gallouët T, Herbin R (2000) Finite volume methods. Handb Numer Anal 7:713-1018

7. LeVeque RJ (2002) Finite volume methods for hyperbolic problems. Cambridge University press, Cambridge

8. He JH (1999) Homotopy perturbation technique. Comput Methods Appl Mech Eng 178:257-262

9. Singh R, Singh S, Wazwaz AM (2016) A modified homotopy perturbation method for singular time dependent EmdenFowler equations with boundary conditions. J Math Chem 54(4):918-931

10. Karunakar P, Chakraverty S (2018) Solution of interval shallow water wave equations using homotopy perturbation method. Eng Comput 34:1610-1624

11. Karunakar P, Chakraverty S (2017) Comparison of solutions of linear and non-linear shallow water wave equations using homotopy perturbation method. Int J Numer Methods Heat Fluid Flow 27:2015-2029

12. Makinde OD, Animasaun IL (2016) Thermophoresis and Brownian motion effects on MHD bioconvection of nanofluid with nonlinear thermal radiation and quartic chemical reaction past an upper horizontal surface of a paraboloid of revolution. J Mol Liq 221:733-743

13. Motsa SS, Animasaun IL (2018) Bivariate spectral quasi-linearisation exploration of heat transfer in the boundary layer flow of micropolar fluid with strongly concentrated particles over a surface at absolute zero due to impulsive. Int J Comput Sci Math 9(5):455-473

14. Makinde OD, Omojola MT, Mahanthesh B, Alao FI, Adegbie KS, Animasaun IL, Wakif A, Sivaraj R, Tshehla MS (2018) Significance of buoyancy, velocity index and thickness of an upper horizontal surface of a paraboloid of revolution: the case of Non-Newtonian carreau fluid. Defect Diffus Forum 387:550-561. https://doi. org/10.4028/www.scientific.net/ddf.387.550

15. Animasaun IL, Mahanthesh B, Koriko OK (2018) On the motion of non-newtonian Eyring-Powell fluid conveying tiny gold particles due to generalized surface slip velocity and Buoyancy. Int J Appl Comput Math 4(6):137

16. Piessens $R$ (2000) Computing integral transforms and solving integral equations using Chebyshev polynomial approximations. J Comput Appl Math 121(1-2):113-124

17. Yuksel G, Sezer MA (2013) Chebyshev series approximation for linear second-order partial differential equations with complicated conditions. Gazi Univ J Sci 26(4):515-525

18. Khalifa AK, Elbarbary EM, Elrazek MA (2003) Chebyshev expansion method for solving second and fourth-order elliptic equations. Appl Math Comput 135(2-3):307-318

19. Akyüz-Dascioglu A (2009) Chebyshev polynomial approximation for high-order partial differential equations with complicated conditions. Numer Methods Partial Differ Equ 25(3):610-621

20. Bhrawy AH, Tharwat MM, Yildirim A (2013) A new formula for fractional integrals of Chebyshev polynomials: application for solving multi-term fractional differential equations. Appl Math Model 37(6):4245-4252

21. Caporale GM, Cerrato M (2010) Using Chebyshev polynomials to approximate partial differential equations. Comput Econ 35(3):235-244

22. Sweilam NH, Nagy AM, El-Sayed AA (2015) Second kind shifted Chebyshev polynomials for solving space fractional order diffusion equation. Chaos, Solitons Fractals 73:141-147

23. Mall S, Chakraverty S (2015) Numerical solution of nonlinear singular initial value problems of Emden-Fowler type using Chebyshev Neural Network method. Neurocomputing 149:975-982

24. Mall S, Chakraverty S (2014) Chebyshev neural network based model for solving Lane-Emden type equations. Appl Math Comput 247:100-114

25. Ding J, Tian HY, Chen CS (2009) The recursive formulation of particular solutions for inhomogeneous elliptic PDEs with Chebyshev basis functions. Commun Comput Phys 5:942-958

26. Ghimire BK, Tian HY, Lamichhane AR (2016) Numerical solutions of elliptic partial differential equations using Chebyshev polynomials. Comput Math Appl 72(4):1042-1054

27. Lamichhane AR, Young DL, Chen CS (2016) Fast method of approximate particular solutions using Chebyshev interpolation. Eng Anal Bound Elem 64:290-294

28. Lin J, Chen CS, Wang F, Dangal T (2017) Method of particular solutions using polynomial basis functions for the simulation of plate bending vibration problems. Appl Math Model 49:452-469

29. Agarwal P, El-Sayed AA (2018) Non-standard finite difference and Chebyshev collocation methods for solving fractional diffusion equation. Phys A 500:40-49

30. Snyder MA (1966) Chebyshev methods in numerical approximation, vol 2. Prentice Hall, Inc., Englewood Cliffs, pp 23-70

31. Tohidi E (2015) Application of Chebyshev collocation method for solving two classes of non-classical parabolic PDEs. Ain Shams Eng J 6:373-379

Publisher's Note Springer Nature remains neutral with regard to jurisdictional claims in published maps and institutional affiliations. 